\title{
Avaliação dos depósitos da pulverização em frutos de cafeeiro utilizando dois equipamentos associados a diferentes volumes de calda
}

\author{
Gustavo Rabelo Botrel Miranda \\ Instituto Federal de Educação, Ciência e Tecnologia Sul de Minas, campus Muzambinho, grbmiranda@gmail.com \\ Carlos Gilberto Raetano \\ Departamento de Produção Vegetal/Defesa Fitossanitária/UNESP/FCA, campus Botucatu, raetano@fca.unesp.br \\ Vilmar Codignoli da Silva \\ Instituto Federal de Educação, Ciência e Tecnologia Sul de Minas, campus Machado, vilmarcodignole@bol.com.br \\ Mateus Delgado Queiroz Cunha \\ Instituto Federal de Educação, Ciência e Tecnologia Sul de Minas, campus Machado, cotonetecafe@gmail.com \\ Renato Humberto de Carvalho \\ Instituto Federal de Educação, Ciênciae Tecnologia Sul de Minas, campus Machado, imagemadesivo@yahoo.com.br \\ Jader Moura Pinheiro \\ Instituto Federal de Educação, Ciência e Tecnologia Sul de Minas, campus Machado, jadermoura@hotmail.com
}

Marcelo de Paiva Gonçalves

Instituto Federal de Educação, Ciência eTecnologia Sul de Minas, campus Machado, marcelo_tecnologocafe@yahoo.com.br

Carlos Henrique Rodrigues Reinato

Instituto Federal de Educação, Ciência e Tecnologia Sul de Minas, campus Machado, carlosreinato@bol.com.br

Leandro Carlos Paiva

Instituto Federal de Educação, Ciência e Tecnologia Sul de Minas, campus Machado, Icpaiva@eafmachado.gov.br

Demétrius de Araújo

Universidade Estadual Paulista “Júlio de Mesquita Filho", campus de Botucatu, demaraujo@yahoo.com.br

\section{Resumo}

Com o objetivo de avaliar a quantidade de depósitos da pulverização nos frutos de café com dois equipamentos associados a diferentes volumes de calda, foi instalado um experimento em lavoura de café (Acaiá) em uma fazenda do município de Campos Gerais (MG). O experimento constou de três volumes de calda (150, 300 e 600 L.ha-1). O primeiro volume foi pulverizado com o pulverizador pneumático Martignani, modelo Whirlwind B612, e os outros dois volumes com o pulverizador de pressão hidráulica convencional. O delineamento foi de blocos casualizados (DBC) com sete repetições. Cada parcela constou de três linhas com dez plantas (4 plantas úteis). As avaliações foram realizadas coletando-se cinco frutos nas partes inferiores e superiores após a pulverização das quatro plantas avaliadas, formando o esquema de parcelas subdivididas no espaço. Os frutos foram lavados e os depósitos quantificados em espectrofotômetro à leitura de $630 \mathrm{~nm}$ no Instituto Federal de Educação, Ciência e Tecnologia do Sul de Minas Gerais (IFSULDEMINAS), campus Machado. Os valores dos depósitos de pulverização foram analisados pelo teste $\mathrm{F}$ e as médias comparadas pelo teste de Tukey a $5 \%$ de significância no programa SISVAR 4.3. Concluiu-se que as aplicações com pulverizador de pressão hidráulica (300 e 600 L/ha) proporcionaram depósitos maiores na parte inferior do cafeeiro.

Palavras-chave: Coffea arabica L., Tecnologia de aplicação, Pulverizador, Corante 


\section{Two equipments associated the different carrier volumes for the evaluation of spray deposit on coffee fruits}

\begin{abstract}
Having the objective of evaluate the quantity of spray deposits on coffee fruits by two equipments with different carrier volumes, the work was carried out on a farming of coffee (Acaiá) located on a farm in the town of Campos Gerais (MG), and it consisted of tree carrier volumes (150, 300 and $600 \mathrm{~L} / \mathrm{ha}$ ), being the first volume pulverized with Martignani turbopulverizator, and the others two with the conventional sprayer for coffee. The treatments were arranged in randomized blocks design (DBC) with seven repetitions. The plot consisted of 3 rows with 10 plants, (4 central plants were evaluated). The evaluations were carried out by collecting 5 fruits in the superior and inferior parts after the spraying of 4 evaluated plants, forming the scheme of subdivided plots in the space. The fruits were washed and the spray deposits quantified by spectrofotometer at $630 \mathrm{~nm}$ at the IFSULDEMINAS Campus de Machado. The values of spray deposits were analyzed by the $\mathrm{F}$ test and the averages were compared by the Tukey test at $5 \%$ of significance in SISVAR 4.3 program. It's concluded that the applications with sprayer with hydraulic energy reached a higher deposit levels in the inferior part of the coffee plant.
\end{abstract}

Keywords: Application technology, Coffea Arabica L., Pulverizator, Color Pigment.

\section{Introdução}

O café Coffea arabica L., planta natural da Etiópia, foi introduzido no Brasil em 1727 com as primeiras mudas sendo plantadas em Belém, no Estado do Pará. Hoje a cultura é largamente plantada em diversas regiões do país e do continente americano, onde o Brasil é o maior produtor mundial com aproximadamente $32 \%$ da produção total (Thomaziello et al., 1997; GUIMARÃES et al., 2002; Agrianual, 2008).

Com o aumento da área cultivada, cria-se ambiente favorável ao desenvolvimento de pragas e doenças, destacando-se entre elas, a ferrugem do cafeeiro (Hemileia vastratix B.B.), a broca-do-cafeeiro (Hypothenemus hampei F.), o bicho mineiro (Leucoptera coffeella G.M.) e os nematóides das galhas (Meloidogyne spp. G.) (Guimarães et al., 2002, Thomaziello, et al. 1997). A ocorrência desses insetos e fitopatógenos na cultura torna necessária a adoção de medidas de controle, com destaque para o uso de produtos fitossanitários (Matuo, 2005).

$\mathrm{Na}$ aplicação desses produtos, a deposição e distribuição do ingrediente ativo na parte aérea da planta dependem de diversos fatores como tamanho da planta, densidade da copa, deriva, tamanho de gota, volume de calda, forma e volume de planta, velocidade de deslocamento do pulverizador, velocidade do vento, tipo de equipamento utilizado, combinação de bicos no pulverizador em relação à planta, volume e velocidade do ar na saída do pulverizador e distância do pulverizador até o alvo (Raetano, 1996).

Para Pezzopane et al. (2003), Ramos et al. (2007), Scudeler et al. (2004) e Tavares Junior et al. (2002), os cultivares de café, o formato da planta, a altura, o comprimento de ramos, a quantidade e tamanho de frutos, a idade e a densidade foliar das plantas podem constituir sérios obstáculos à penetração de produtos fitossanitários em forma de gotas.

Desta forma, o desenvolvimento de novas tecnologias e/ou adaptações de técnicas de aplicações já utilizadas em outras culturas, como a dos citros, podem fornecer à cafeicultura boas alternativas com alta eficiência de pulverização, como é o caso do pulverizador pneumático Martignani, modelo Whirlwind B612 "Autonom-Trac", com formação de gotas por energia gasosa através dos bicos tipo 'UGELLI' - "pulverizador pneumático" (Martignani, 2008). Matuo (1990) define a formação de gotas por energia gasosa quando o fluxo de ar fragmenta o fluxo líquido em gotas.

Outro fator que influencia na eficiência de uma pulverização está relacionada ao volume de aplicação, o qual vem sendo objeto de muitos estudos na área da tecnologia de aplicação. Porém, com volumes de calda reduzidos, a uniformidade na distribuição de gotas tornase um objetivo a ser alcançado para manter a eficiência do controle. Por isso, a uniformidade de deposição do líquido pulverizado e o tamanho das gotas são fatores que dependem do desempenho do órgão emissor de gotas que, no caso dos pulverizadores, é a ponta hidráulica, considerada a parte mais importante do equipamento (Bauer; Raetano, 2004).

O objetivo deste trabalho foi avaliar os depósitos da pulverização em frutos do cafeeiro, através do uso de uma substância marcadora, aplicada por dois pulverizadores associados a três volumes de calda (150, 300, 600 L.ha' $\left.{ }^{-1}\right)$ utilizados na cultura do café.

\section{Material e métodos}

O experimento foi instalado numa lavoura de café, cultivar Acaiá, em plantio convencional com espaçamento de 3,6 $\mathrm{x}$ 0,9 m, 10 anos de idade, conduzida com 2,35 metros de altura, podada anualmente, localizada no município de Campos Gerais, Minas Gerais, com uma altitude média de $815 \mathrm{~m}$.

O delineamento experimental foi de blocos ao acaso (DBC), com 7 repetições para 3 volumes de calda, sendo o volume menor (150 L.ha') aplicado com pulverizador pneumático e os volumes de 300 e 600 L.ha" de calda, com pulverizador de pressão hidráulica. As parcelas, 
constituídas por três linhas de plantio com dez plantas cada, foram pulverizadas e foram avaliadas as quatro plantas centrais. As demais linhas e plantas foram consideradas como bordadura.

Foi utilizado um pulverizador pneumático Martignani, modelo Whirlwind B612 "Autonom-Trac", dotado de um tanque em aço esmaltado (Aço - Stainless Steel) com bomba centrífuga de grande capacidade (250 L. $\left.\mathrm{min}^{-1}\right)$, com selos mecânicos, ventilador centrífugo com dupla aspiração e desempenho aerodinâmico alto $\left(25.000 \mathrm{~m}^{3} \cdot \mathrm{h}^{-1}\right.$ de ar a $\left.80 \mathrm{~m} . \mathrm{s}^{-1}\right)$, comando remoto fluido tipo mecânica, distribuidor de graduação em aço inox para todos os volumes, quatro difusores de $90^{\circ}$ contendo seis bicos em cada difusor (bicos "Ugelli", com largura do passo de 4 mm de diâmetro) (Martignani, 2008) totalizando 24 bicos, a fim de realizar a pulverização com 150 L.ha'-1. A pressão de trabalho do pulverizador pneumático foi de $123,56 \mathrm{kPa}$, sendo a velocidade do vento da turbina BWM B612 equivalente a $60 \mathrm{~m} \cdot \mathrm{s}^{-1}$, conforme informações do fabricante.

Utilizou-se o pulverizador de pressão hidráulica modelo ARBUS 1000 com ventilador axial que proporciona ventilação equivalente a $42 \mathrm{~m} . \mathrm{s}^{-1}$ para realizar a pulverização dos volumes de 300 e 600 L.ha', com bicos de jato cônico vazio modelo JA-1 para 300 L.ha $^{-1}$ e JA-2 para
600 L.ha'. O pulverizador possui um ramal de sete saídas duplas em cada lado do pulverizador, sendo utilizadas para as pulverizações somente seis saídas de cada lado, totalizando vinte e quatro saídas para bicos de pulverização, a uma pressão de 455,03 kPa (Tabela 1).

Os dados meteorológicos (temperatura, umidade relativa do ar e velocidade do vento) durante a aplicação da calda de pulverização foram registrados por um termohigrômetro (Modelo HT-3003) e anemômetro (Modelo AM-4201) (Tabela 2), ambos da marca Lutron, posicionados a dois metros de altura da superfície do solo (Scudeler et al., 2004).

Para quantificar os depósitos da pulverização com os respectivos volumes de calda, foi utilizada uma substância marcadora: corante alimentício Azul Brilhante (FD\&C $n^{\circ} 1$ ) à concentração de $0,15 \%(\mathrm{p} / \mathrm{v})$, para que, após a pulverização, fossem colhidas duas amostras de cinco frutos por planta avaliada, uma amostra na parte superior (1,75 m de altura) e outra amostra na parte inferior da planta (1,00 $\mathrm{m}$ de altura). Os frutos foram colhidos aleatoriamente dos dois lados da planta, na região de produção dos frutos de café: interior da copa do cafeeiro, já que é nessa região que acontece a frutificação do café

$\mathrm{Na}$ colheita dos frutos foram utilizadas luvas de procedimento cirúrgico e pinça, lavadas e secas entre uma

\begin{tabular}{|c|c|c|c|c|c|c|c|}
\hline Tratamento & $\begin{array}{l}\text { Equipamento } \\
\text { (Pulverizador) }\end{array}$ & Bicos & $\begin{array}{c}\text { Tipo } \\
\text { de bicos }\end{array}$ & $\begin{array}{c}\text { Pressão } \\
\text { kPa }\end{array}$ & $\begin{array}{c}\text { Velocidade } \\
\text { (trator + } \\
\text { pulverizador) }\end{array}$ & $\begin{array}{c}\text { Vol. de } \\
\text { aplicação } \\
\text { L.ha }^{-1}\end{array}$ & $\begin{array}{c}\text { Vazão } \\
\text { por bico } \\
\text { L.min }\end{array}$ \\
\hline 1 & Pneumático* & 24 & $\begin{array}{l}\text { Jato de ar } \\
\text { (UGELLI) }\end{array}$ & 126,56 & $\begin{array}{c}5,14 \mathrm{~km} \cdot \mathrm{h}^{-1} \\
1800 \mathrm{rpm} \\
\left(4^{\underline{a}} \text { reduzida }\right)\end{array}$ & 150 & 0,193 \\
\hline 2 & $\begin{array}{l}\text { Hidráulico } \\
\text { Arbus } 1000\end{array}$ & 24 & $J A-1$ & 455,03 & $\begin{array}{c}4,73 \mathrm{~km} \cdot \mathrm{h}^{-1} \\
1700 \mathrm{rpm} \\
\left(4^{\underline{a}} \text { reduzida }\right)\end{array}$ & 300 & 0,355 \\
\hline 3 & $\begin{array}{l}\text { Hidráulico } \\
\text { Arbus } 1000\end{array}$ & 24 & $J A-2$ & 455,03 & $\begin{array}{c}3,83 \mathrm{~km} \cdot \mathrm{h}^{-1} \\
1700 \mathrm{rpm} \\
\left(3^{\underline{a}} \text { reduzida) }\right.\end{array}$ & 600 & 0,575 \\
\hline
\end{tabular}

Tabela 1: Equipamentos e condições operacionais na aplicação da solução contendo o corante Azul Brilhante para avaliação dos depósitos da pulverização em frutos de café. * Pulverizador pneumático Martignani modelo Whirlwind B612 "Autonom-Trac".

\begin{tabular}{|c|c|c|c|c|}
\hline Blocos & $\begin{array}{c}\text { Data de } \\
\text { pulverização }\end{array}$ & $\begin{array}{c}\text { Temperatura } \\
\left({ }^{\circ} \mathrm{C}\right)\end{array}$ & $\begin{array}{c}\text { Umidade } \\
\text { Relativa (\%) }\end{array}$ & 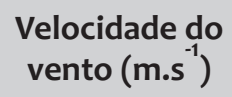 \\
\hline 1 & 5 de março & 27,4 a 33,2 & 32 a 57 & 3,96 a 4,7 \\
\hline 2 e 3 & 6 de março & 27,7 a 30,7 & 42 a 58 & 0,9 a 7,2 \\
\hline 4 & 7 de março & 25,4 a 32,2 & 43 a 66 & o a 10,9 \\
\hline
\end{tabular}

Tabela 2: Temperatura, umidade relativa e velocidade do vento em cada dia durante as pulverizaçoes realizadas em cafeeiro. 
colheita e outra, para transferir os frutos a sacos plásticos ( 5 frutos/saco) identificados a fim de não ocorrer perda do corante marcador entre colheitas, bem como evitar contaminação entre uma amostra e outra. Tal procedimento também foi adotado na análise em laboratório.

As amostras foram conduzidas ao laboratório de análises químicas de café do Instituto Federal de Educação, Ciência e Tecnologia do Sul de Minas Gerais (IFSULDEMINAS), campus de Machado, e a cada amostra foram adicionados $20 \mathrm{~mL}$ de água destilada, mantendo-a sob agitação por 30 s para a remoção do corante. A solução resultante foi colocada em frascos de vidro e mantida sob refrigeração $\left(8 \pm 3^{\circ} \mathrm{C}\right)$. Após este procedimento, os depósitos foram quantificados por um espectrofotômetro marca Femto e procedeu-se a leitura dos valores de absorbância no comprimento de onda de $630 \mathrm{~nm}$ e calculou-se a área da superfície dos frutos coletados.

As leituras das amostras foram ajustadas através do estabelecimento de curva-padrão a partir das seguintes diluições: 1:50, 1:100, 1:500, 1:1000, 1:2000 e 1:10000 de cada calda de pulverização contendo o corante marcador coletada no campo. Em seguida, fizeram-se as leituras de

\section{Curva de concentração padrão}

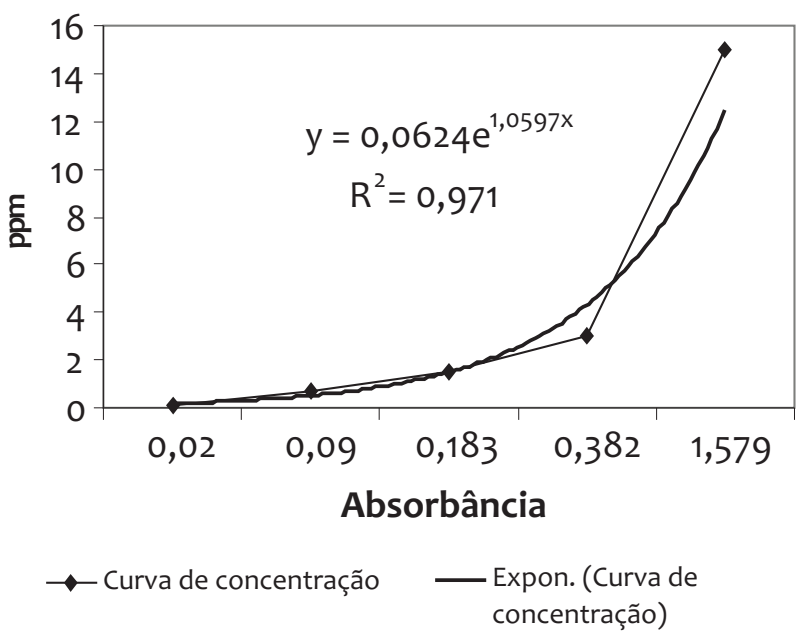

Figura 1: Curva da leitura de absorbância para ajuste da concentração das amostras dos respectivos volumes de calda. absorbância das amostras em espectrofotômetro, possibilitando a transformação dos valores em ppm (mg. $\mathrm{L}^{-1}$ ) e ajustando-os à curva previamente definida.

A quantificação do corante nas caldas possibilitou o estabelecimento da equação 1 para todos os volumes de calda (Figura 1):

$$
y=0,0624 e^{1,0597 x}
$$

Sendo $\mathrm{x}$ = leitura da densidade óptica (absorbância), y = a concentração em ppm (mg. $\left.\mathrm{L}^{-1}\right)$.

Para calcular o volume de calda depositado no fruto, mediram-se, inicialmente, os três diâmetros do grão do café; dois transversais e um longitudinal - para extrair a média dos diâmetros e assim considerar o fruto como uma esfera equivalente, calculando a área externa do fruto, utilizando o diâmetro médio através da equação 2.

$$
S=\pi \cdot d^{2}
$$

em que:

$\mathrm{S}=$ área da superfície do fruto $\left(\mathrm{mm}^{2}\right)$;

$\pi=3,14$;

$\mathrm{d}=$ média dos diâmetros do fruto $(\mathrm{mm})$.

Em seguida, determinou-se o volume de calda depositado no fruto a partir da leitura de absorbância obtida da solução de lavagem dos frutos, através da equação 3:

$$
C 1 \cdot V 1=C 2 \cdot V 2 \quad[3]
$$

onde:

C1 = Concentração inicial do corante marcador na calda, neste caso, $1500 \mathrm{ppm}$;

V1 = Volume inicial, neste caso o volume a ser calculado;

C2 = Concentração final correspondente à concentração encontrada na leitura do espectrofotômetro;

$V_{2}=$ Volume final, neste caso o volume utilizado para lavar

\begin{tabular}{|c|c|c|c|}
\hline \multirow{2}{*}{$\begin{array}{c}\text { PULVERIZADOR/ } \\
\text { VOLUME DE CALDA }\end{array}$} & \multicolumn{3}{|c|}{ Depósito da pulverização ( $\left.\mu \mathrm{L} . \mathrm{cm}^{-2}\right)$} \\
\hline & $\begin{array}{l}\text { Parte Superior } \\
\text { da planta }\end{array}$ & $\begin{array}{l}\text { Parte Inferior } \\
\text { da planta }\end{array}$ & Média \\
\hline Pneumático/150 L & 0,1373 a & 0,1437 a & $0,1405 \mathrm{~A}$ \\
\hline Hidráulico/300 L & 0,1479 a & $0,1612 b$ & $0,1545 B$ \\
\hline Hidráulico/60o L & 0,1513 a & $0,1617 b$ & $0,1565 \mathrm{~B}$ \\
\hline Média & 0,1455 a & $0,1555 b$ & 0,1505 \\
\hline
\end{tabular}
osfrutos.

Após determinar o volume da pulverização nos

Tabela 3: Depósitos de pulverização ( $\mu \mathrm{L} . \mathrm{cm}-2)$ com diferentes combinações entre pulverizador e volume de calda em frutos de café. $C V=$ Acaiá. Safra= 2007/2008. Campos Gerais/MG. Médias seguidas pela mesma letra minúscula na linha e maiúscula na coluna não se diferem entre si pelo teste de Tukey a $5 \%$ de significância. 
frutos, calculou-se o depósito em microlitros de calda por área de fruto $\left(\mathrm{cm}^{2}\right)$. Os dados obtidos foram submetidos à análise de variância pelo teste $\mathrm{F}$ em esquema de parcela subdividida no espaço e as médias foram comparadas pelo teste de Tukey ao nível de $5 \%$ de probabilidade realizada pelo programa SISVAR 4.3 (Ferreira, 2000).

\section{Resultados e discussão}

As médias dos depósitos da solução contendo corante por área de frutos do cafeeiro nos respectivos tratamentos estão apresentados na Tabela 3.

Os valores médios dos volumes de calda de 300 e 600 L.ha' ${ }^{-1}$ pulverizados com o pulverizador hidráulico proporcionaram maior deposição nos frutos de cafeeiro. Porém, foi observada maior deposição na parte inferior do cafeeiro que, apesar da quantidade de folhas nessa parte da planta ser visualmente maior que na parte superior, não houve diferença para a parte superior da planta. Os bicos do pulverizador hidráulico trabalham mais próximos dessa região da planta em relação à parte superior, justificando os resultados observados.

Scudeler et al. (2004) verificaram, com uso de corantes, que o volume de calda atingido na parte inferior do cafeeiro é maior que o volume de calda atingido na parte superior das plantas e os autores atribuíram isso a uma deficiência no equipamento de tração mecânica para pulverização.

Ramos et al. (2007) sugerem a possibilidade de levantar o conjunto ventilador e bicos até a altura média do cafeeiro para aproximar os bicos superiores ao ápice da planta e distribuir melhor as gotas para evitar tal problema, quando se tratar de pulverizadores montados.

Raetano et al. (2001), avaliando a deposição da pulverização com diferentes equipamentos em cafeeiros do cultivar catuaí (porte baixo), verificaram maior dificuldade de penetração das gotas e cobertura dos frutos da parte inferior da planta. Esses resultados podem ter divergido dos encontrados neste trabalho devido ao porte mais alto do cultivar Acaiá, que proporciona maior distância dos bicos superiores do pulverizador até os ramos plagiotrópicos superiores do cafeeiro, quando comparado à distância entre bicos superiores e ramos plagiotrópicos de um cultivar de porte baixo (Catuaí). Essa distância maior entre os bicos mais altos do pulverizador e os ramos superiores da planta pode dificultar a deposição das gotas nessa região. Esse fato pode justificar a ineficiência da deposição em frutos mais altos do cafeeiro quando utilizado o pulverizador de pressão hidráulica mesmo com volumes maiores, já que o sistema de assistência de ar do pulverizador de pressão hidráulica proporciona ventos inferiores ao pulverizador pneumático. Outro ponto importante são os internódios de cultivares de café de porte alto, que são maiores quando comparado aos de porte baixo. Tal fato, no cultivar Acaiá, pode facilitar a entrada das gotas de pulverização nos ramos baixeiros que estão mais próximos dos bicos do pulverizador, também com internódios maiores.

Os valores médios dos depósitos de calda por área de frutos obtidos realizada com o pulverizador pneumático com 150 L.ha' ${ }^{-1}$ foi menor em relação aos outros volumes de calda pulverizados com o pulverizador hidráulico. Porém, não foi observada diferença nos depósitos avaliados em partes da planta para a pulverização deste volume/equipamento (150 L.ha-1- pneumático)e, por isso, a pulverização foi mais homogênea que os outros volumes de calda pulverizados pelo pulverizador hidráulico (Tabela 3).

Ainda é importante comentar que a velocidade de deslocamento do conjunto trator + pulverizador pode interferir negativamente nos resultados de deposição em frutos ou folhas de plantas. Porém, acredita-se que para este trabalho a influência da velocidade não interferiu nos resultados obtidos, e que a diferença de deposição nas partes da planta foi mesmo referente aos equipamentos utilizados, pois a diferença de velocidade do pulverizador hidráulico com maior volume de calda (60oL.ha'), $4,73 \mathrm{~km} . \mathrm{h}^{-1}$, foi muito semelhante ao pulverizador pneumático com menor volume de calda (150L.ha ${ }^{-1}$ ), $5,14 \mathrm{~km} . \mathrm{h}^{-1}$. Já que os resultados observados para os tratamentos envolvendo o pulverizador hidráulico foram iguais entre si.

Apesar do pulverizador pneumático ter a mesma quantidade de bicos que o pulverizador hidráulico, o pneumático proporciona melhor deposição das gotas devido à altura dos difusores superiores ser maior que o bico mais alto do pulverizador hidráulico, convencionalmente utilizado em cafeeiro (Martignani, 2008). Com isso, a pulverização dos frutos superiores da planta do cafeeiro são mais atingidos pelos difusores superiores do pulverizador pneumático.

\section{Conclusões}

a) As aplicações com pulverizador hidráulico proporcionam maiores depósitos na parte inferior que na parte superior do cafeeiro.

b) A aplicação com pulverizador pneumático foi mais homogênea que as aplicações com pulverizador hidráulico.

c) O volume de calda em aplicações no cafeeiro pode ser reduzido se utilizado o equipamento pulverizador pneumático, porém a eficiência no controle fitossanitário com volumes menores requer mais estudos.

\section{Agradecimentos}

Agradeço a Deus, aos professores, amigos e funcionários 
da Faculdade de Ciências Agronômicas da Universidade Estadual Paulista "Júlio de Mesquita Filho", campus de Botucatu.

Aos professores, funcionários e alunos do Instituto Federal Sul de Minas, campus de Machado.

\section{Referências bibliográficas}

AGRIANUAL 2008. Café: Balanço mundial. Anuário da Agricultura Brasileira, São Paulo, 2007, p.160.

BAUER, F.C.; RAETANO, C.G. Perfis de distribuição volumétrica de pontas XR 11003 e TXVK-4 em diferentes condições de pulverização. Engenharia Agrícola, Jaboticabal, v.24, n.2, 2004.

FERREIRA, D.R. Análises estatísticas por meio do Sisvar para Windows versão 4.0. In: Reunião Anual da Região Brasileira da Sociedade Internacional de Biometria, 45, 2000, São Carlos. Anais. São Carlos: UFSCar, 2000. p.255258.

GUIMARÃES, R.J.; MENDES, A.N.G.; SOUZA, C.A.S. Cafeicultura. Lavras: UFLA/FAEPE, 2002.317p.

MATUO, T. K. Desenvolvimento de equipamento motorizado para aplicação líquida de agrotóxicos na cultura do café. 2005, 78p. Tese (Doutorado em Agronomia - Pós-graduação em Proteção de Plantas) Faculdade de Ciências Agronômicas - Universidade Estadual Paulista. Botucatu.

MATUO, T. Técnicas de aplicação de defensivos agrícolas. Jaboticabal, São Paulo. Funep, 2 ${ }^{\mathrm{a}}$ edição. 139p. 1990.

MARTIGNANI. Nebulizador neumático-lectrostático "martignani kwh" - modelo whirlwind b612 "a u t o nom trac". Disponível e m : <http://www.martignani.com/pdf/eng/sprayer_b612_aut onomtrac_EN_revised.pdf>Acesso em:18/11/2008.

PEZZOPANE，J.R.M.; PEDRO JÚNIOR, M.J.; THOMAZIELLO, R.A.; CAMARGO, M.B.P.. Escala para avaliação de estádios fenológicos do cafeeiro arábica. Bragantia, Campinas, v.62, n. 3, 2003.

RAETANO, C. G. Condições operacionais de turboatomizadores na distribuição e deposição da pulverização em citros. 1996. 93p. Tese/Doutorado em Agronomia na Universidade de São Paulo USP/Escola Superior de Agronomia Luíz de Queiroz (ESALQ). Piracicaba.

RAETANO, C.G.; SCUDELER, F.; BAUER, F.C.; VENEGAS, F.; VINCHI, R.R.. Avaliação da cobertura de pulverização com diferentes equipamentos e condições operacionais na cultura do café. In: Simpósio Internacional de Tecnologia de Aplicação de Agroquímicos: Eficiência, Economia e Preservação da Saúde Humana e do Ambiente - SINTAG, 2, Jundiaí, 17 a 20 de julho, 2001. Disponível em: < http://www.iac.sp.gov.br/Centros/centro\%20de\%2oengen haria\%20e\%20automação/sintag/anais.htm>.

RAMOS, H. H.; YANAI, K; ARAÚJO, D.; AGUIAR, V. C.. Tecnologia de aplicação de defensivos na cultura do café. In: VII Curso de atualização em café. 2007. Campinas, SP. Anais. Campinas, SP. Coordenadores: Thomaziello, R.A., Fazuoli, L.C.. 91p. (Documentos IAC, 80) Instituto Agronômico, 2007.

SCUDELER, F.; RAETANO, C. G.; ARAUJO, D.; BAUER, F. C. Cobertura da pulverização e maturação de frutos do cafeeiro com ethephon em diferentes condições operacionais. Bragantia, Campinas, v.63, n. 1, 2004.

TAVARES JÚNIOR, J.E.; FAVARIN, J.L.; DOURADO NETO, D.; MAIA, A.H.N.; FAZUOLI, L. C.; BERNARDES, M.S.. Análise comparativa de métodos de estimativa de área foliar em cafeeiro. Bragantia, Campinas, v.61, n. 2, 2002.

THOMAZIELLO, R.A.; OLIVEIRA, E.G.; TOLEDO FILHO, J.A.; COSTA, T.E.. Cultura do Café. Campinas, Coordenadoria de Assistência Técnica Integral CATI, 1997-3 $3^{\text {a }}$ edição. 75p. ilus. 21,5 cm (Boletim Técnico, 193). 